\title{
Distribution of ocular dominance and effect of image clarity*
}

\author{
PATRICIA A. ONDERCIN, N. W. PERRY, JR., and D. G. CHILDERS \\ Visual Sciences Laboraton', University' of Florida, Gainesille, Florida 32601
}

\begin{abstract}
The purposes of this study were to determine if ocular dominance could be measured and defined in a quantitative and continuous manner, rather than dichotomously, and whether such a measure could be related to image sharpness, or acuity. Ocular dominance was assessed on a dichoptic, but nonrivalry, task for $56 \mathrm{Ss}$, who were then assigned to groups according to their degree of dominance. Four positive spherical lenses $(+0.75,+1.25,+1.75,+2.25)$ were used to induce differing amounts of refractive error in one eye. Primary results indicate that (1) dominance, as measured, is a continuous function which is normally distributed in the general population, (2) blurring the image in the dominant eye reduces the degree of dominance, and (3) blurring the image to a nonpreferred eye increases the amount of dominance in the other eye.
\end{abstract}

The human visual system has the ability to fuse two slightly disparate images into a single binocular perception. When the information from one eye is given a functional preference in that percept, ocular dominance is ascribed to that eye. Evidence in the literature is equivocal regarding whether or not dominance is a unitary function. For example, Berner and Berner (1953) make a distinction between the dominant eye. which directs vision in a monocular task such as sighting. and the controlling eye, which takes the primary role in binocular tasks such as reading. Two different types of dominance are also postulated by Walls (1951), i.e., motor and perceptual-sensory, while five kinds of dominance are delineated by Lederer (1961).

Methods used to assess dominance are diverse and reflect the conceptual differences. When Gronwall and Sampson (1971) performed correlations on 18 tests assembled for their battery. they found a nuclear group of five tests with high intercorrelations; a peripheral group moderately correlated with the nuclear group, but highly correlated with one or two similar tests; and a third group, which did not correlate with any other tests. Statistical support was not found for either Walls' (1951) or Lederer's (1961) classifications. Thus. it is unclear whether the tests are measuring different functions or the same function with different degrees of accuracy.

Most of the different dominance tests yield dichotomous descriptions of dominance. It seems likely: that there is a variation in the degree of dominance between Ss to which these measures are relatively insensitive. Perry and Childers (1972) describe a measure which assesses ocular dominance quantitatively. In their

\footnotetext{
*This study is based on a thesis submitted by the first author to the Department of Psychology of the University of Florida in partial fulfillment of the requirements for the degree of Master of Arts. This research was supported in part by Grant EY 00077 and Grant EY 00581 from the National Institutes of Health.
}

dichoptic stimulation method, a different letter is presented briefly and simultaneously to each eye, resulting in a percept of two letters fused. From the S's report of what letters he sees with repeated stimulation. it is possible to calculate the amount of information perceived from each eye, i.e., degree of dominance.

Acuity, or sharpness of the image, would be a logical factor influencing ocular dominance in the binocular perception. but a variety of studies attempting to correlate acuity and dominance have produced contradictory results (Gahagan, 1933; Merrell, 1957: Crovitz, 1961; Adler \& Tal, 1966). These equivocal findings regarding the role of acuity may, in part, result from the conceptual confusion surrounding "dominance" and the low intercorrelation between measures. It was the purpose of this study to examine the relationship between ocular dominance and the sharpness of the image by quantitatively assessing' the effect on dominance of systematically varying the clarity of the stimulus image.

\section{METHOD \\ Subjects}

The Ss were 56 students at the University of Florida. with $20 / 20$ vision or better in each eye.

\section{Apparatus}

A Bausch and Lomb modified Ortho-Rater was used to assess visual acuity. In the dichoptic stimulation test. an American Optical Co. troposcope. modified to accommodate two Massey Dickenson electroluminescent panels. was used to present the stimuli. The panels consist of 14 elements which can be programmed to generate a display of IBMI-format letters. Stimuli were presented foveally for a duration of $50 \mathrm{msec}$ at the rate of one every $4 \mathrm{sec}$. The stimuli appeared as fused in a small dark rectangle. subtending $a$ visual angle of 2 deg $28 \mathrm{~min} \times 2$ deg $56 \mathrm{~min}$. in a 20 -deg white (tungsten) surround. A central fixation point facilitated fusion. The duration. intensity. and particular pattern of the stimuli were programmed by a paper-tape block reader. 
Table 1

\begin{tabular}{|c|c|c|c|}
\hline Category & Group & Experimental Condition & $\mathrm{s}$ \\
\hline Sondominant & $\begin{array}{c}1 \\
11\end{array}$ & $\begin{array}{l}\text { Lens in ese with lower recall scores } \\
\text { Lens in sye with higher recall scores }\end{array}$ & $\begin{array}{l}17 \\
19\end{array}$ \\
\hline Dominant & $\begin{array}{l}111 \\
11\end{array}$ & $\begin{array}{l}\text { Lens in ele "ith lower recall scores } \\
\text { Lens in eye with higher recall scores } \\
\text { (Dominant eye) }\end{array}$ & $\begin{array}{l}10 \\
10\end{array}$ \\
\hline
\end{tabular}

\section{Preliminary Procedure}

Visual acuity was assessed. both binocularly and monocularly for each eve. after which dominance was measured. using the dichoptic recill nreasure of dominance (essentially that of Perry \& Childers. 1972). Pairs of letters 11 ere presented in a modified troposcope so that a different letter was presented to each eye (dichoptic presentation). the percept being the fusion of the two letters. Pilot work indicated that the measure was highly reliable ir $=+80$ ). All letters were first presented twice binocularis (the same letter to both eyes) in order to familiarize the $S$ with the configurations. To evaluate ocular dominance. 96 letter pairs were presented dichoptically. with a 4 -sec intertrial interval to minimize short-term memory effects. After each trial. the $S$ reported the letter or letters seen. Within the test. each pair was reversed as to eye laterality to ensure equal brightness and equal ditticultr between eres.

Diwhoptic dominance scores were calculated as difference cores. D T. where D equals the difference between the number of letters correctly recalled in the right eye and the number correctly recalled in the left eye. and $\mathrm{T}$ equals the total number of letters correctly recalled in both eyes. On the basis of these scores. Ss were divided into two groups: dominant. or strong preference for one ere: and nondominant, or no strong preference for eirher ele. Pilor work suggested this division as the point where the difference in scores between ey es was 20 r of the total. If $\mathrm{D} T>20$, the $\mathrm{S}$ was classified dominant: if $D T \leqslant 20$. the $S$ was classified nondominant.

\section{Experimental Procedure}

Under the experimental condition. positive spherical lenses "iere introduced to reduce image clarity in one eye during dichoptic stimulation. Ss were assigned randomly to one of four groups. which determined the eye to be blurred (see Table 1).

Each $S$ was tested with each of four positive spherical lenses of different dioptric power: $+0.75+1.25 .+1.75 .+25$. For each lens. a different dichoptic stimulation test of 26 letter pairs was administered. The order in which lenses were used was iaried randomly between $S$ s to control for serial effects. Percentage scores of the number of correct identifications out of the total number of letters presented were calculated for each eve. blurred and nonblurred.

To determine the Ss' ability to discriminate stimuli under blurred conditions independent of dominance. an additional monocular test was given. The eye that did not receive the lens in the experimental condition was occluded. and 20 single letters were presented. blurred by the most powerfiul lens $(+2.25)$. to the other eve. The 15 Ss who could not recognize $90 \%$ of the stimuli were dropped from the experiment.

\section{RESULTS}

Eye dominance, as measured by dichoptic recall. was distributed normally in the sample in direction (right or left eye) and degree (see Fig. 1). The majority of Ss were clustered around the mean. indicating no strong preference for either eye. Strongly dominant Ss are represented at the extremes of the distribution ( $|D / T|$ $>.20$ ) and account for slightly over one-third of the sample.

The differential effects of reducing image clarity on the recall scores for the four groups of $S s$ are shown in Fig. 2. There were no statistically significant differences. when tested with a test between means. between Groups I and II (no preferred eye) under any lens condition: scores were nearly identical whether the blurred eye had the higher or lower recall score. In contrast. there were significant differences $(p<.005)$ between Groups III and IV (which had a strongly preterred eye), dependent upon whether the blurred eye had the higher or lower recall score when the weaker lenses were used $(+0.75 .+1.25)$. The two most powerful lenses $(+1.75$ and +2.25$)$ could eliminate differences in scores between a dominant and a nondominant eye. More complex statistical analyses were performed on the data presented in Fig 2 (and on that presented in subsequent figures). but did not add to the marked effects apparent in the simple graphic representations.

There was a striking difference in percentage scores under monocular and dichoptic conditions (Fig. 3). All groups were capable of scoring $90 \%$ correct or better when a monocular stimulus was blurred with the +2.25 lens. Under dichoptic conditions with the same lens

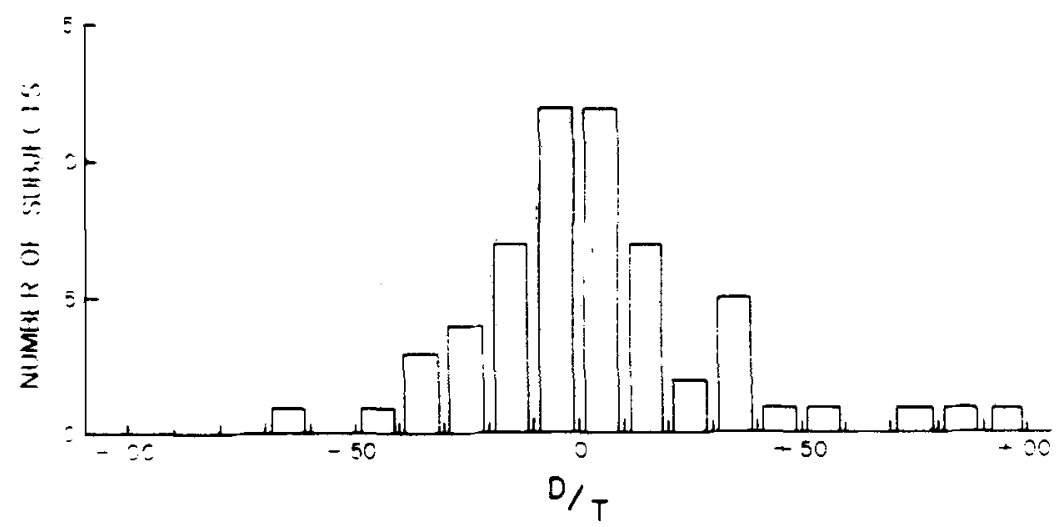

Fig. 1. Distribution of degree and direction of dominance in the $S$ population. - $D / T$ on the abscissa is the difference between the number of letters correctly recalled from each eye. divided by the total correctly recalled from both eyes. 
Fig. 2. Recall scores vs experimental condition for all four experimental groups. Open circles, Group I with lens in the eye with lower recall scores on the initial dominance test: filled circles, Group II with lens in the eye with higher recall scores: Ss in both groups demonstrated no strong preference for either eye in the "no-lens" condition. Open squares, Group Ill with lens in the eye with lower recall scores (nondominant eye); filled squares. Group IV with lens in the eye with higher recall scores (dominant eye): Ss in both groups demonstrated a very strong preference for one eye over the other in the "no-lens" condition.

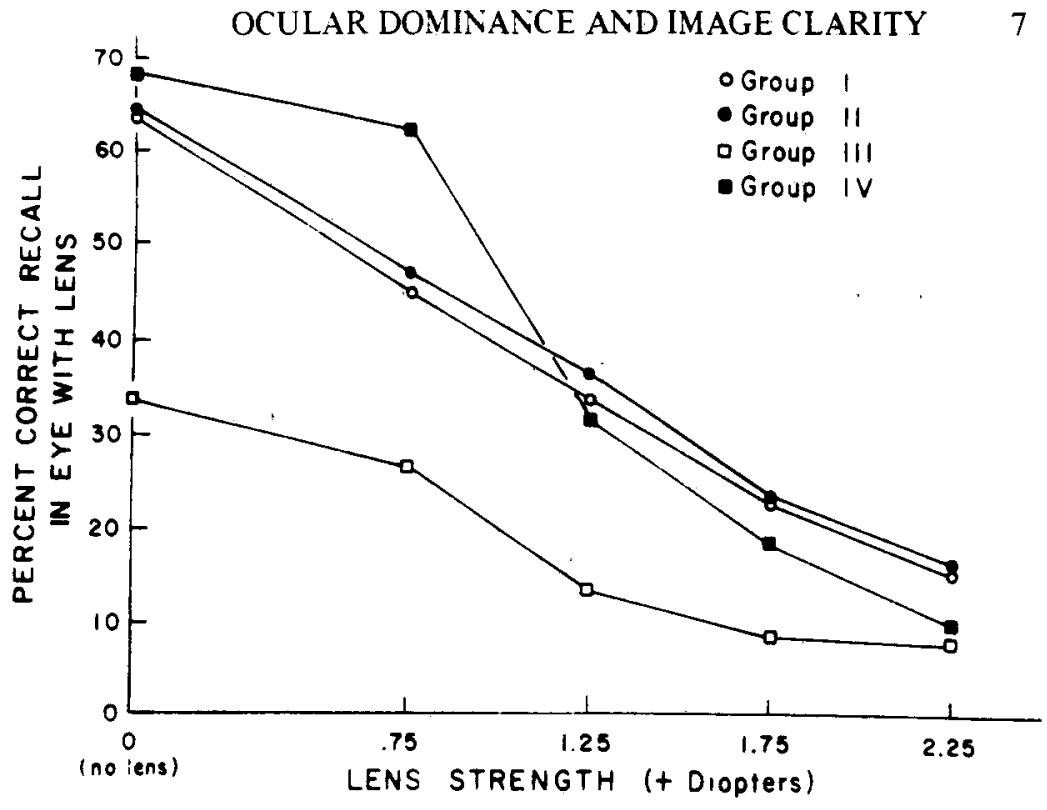

blurring one eye, recall in that eye dropped to less than $20 \%$.

Figure 4 depicts changes in scores from the nonblurred eye concomitant with increasing lens strength in the other eye, and is the mirror image of Fig. 2. The introduction of the +1.75 lens in the other eye markedly increased scores in the nonblurred eye. For Groups I. II, and III, the curves are almost flat; the least powerful lens $(+0.75)$ in the opposite eye enhanced scores in the nonblurred eye nearly as much as did the most powerful $(+2.25)$. In Group IV, in which the dominant eye was being blurred, a more powerful lens $(+1.75)$ was required to bring the performance of the nonpreferred eye up to the level reached in the other groups.

\section{DISCUSSION}

The finding that the amount of dominance approximates a normal distribution confirms, with a large normal sample, the report of Perry and Childers (1972). Thus, designation of dominant and nondominant individuals by artificially dichotomizing a continuous distribution may yield groups different from those designated from dichoptic measures. Further, since most individuals fall in the center of the distribution, a very small measurement error would shift them from one to the other eye with a dichotomous dominance measure.

The results indicate that ocular dominance can be established. enhanced. or decreased by the reduction of image clarity in one eve. The differential effect of the lenses on recall scores indicates that the amount of change which occurs is dependent on both the amount of blurring introduced and the baseline dominance of the eye. Dominance of one eye and relative suppression of the other eye can be easily established in Ss without a preferred eye. In Ss who have a strong priority of one

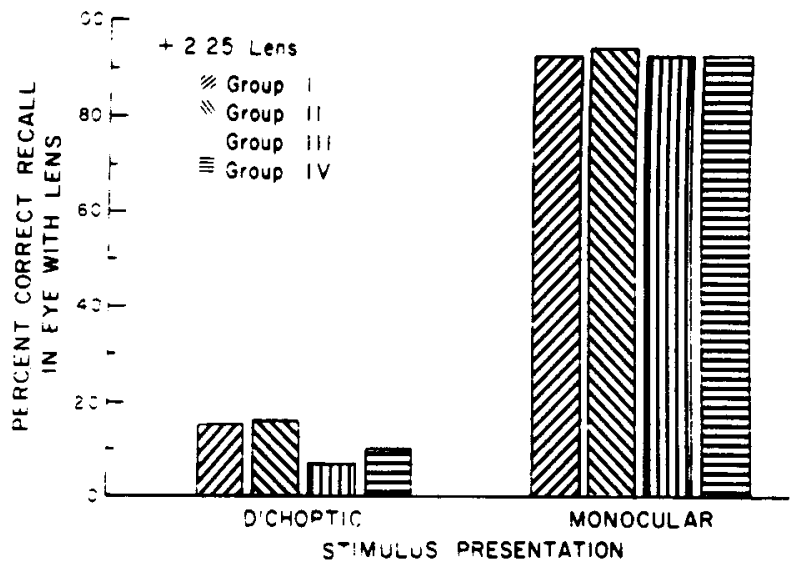

Fig. 3. Comparison between monocular and dichoptic performance under the +2.25 lens condition for all groups. Group designations same as for Fig. 2.

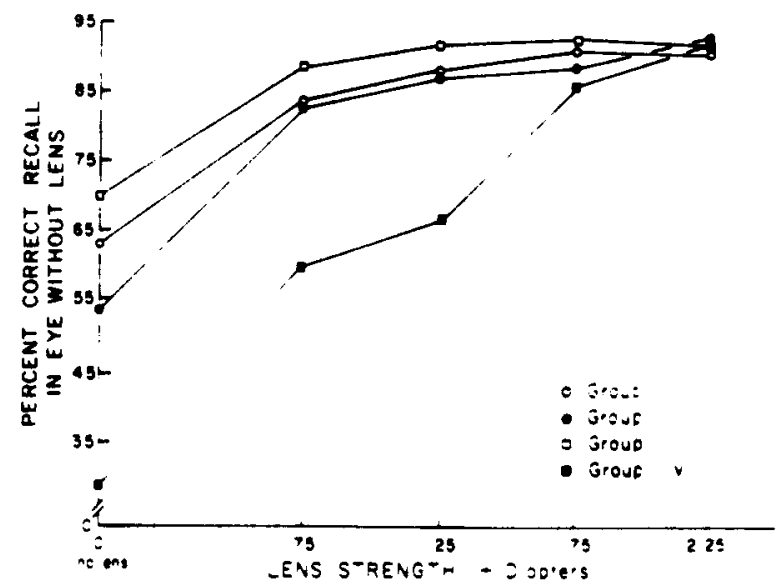

Fig. 4. Recall scores from the nonblurred eye during dichoptic stimulation. Abscissa gives strength of lens in the other eye. Group designations same as for Fig. 2. 
eye. it is easier to enhance preexisting tendencies than to reverse them, but it is possible to do both. Dominance. as measured by dichoptic stimulation. is not a static characteristic even in adults: dominance was shifted in even strongly dominant $S$ s.

Under monocular conditions. when the image is blurred by the most powerful lens $(+2.25)$. Ss in all four groups scored better than $90^{\circ}$ correct. It follows. then. that the decrement in scores which accompanies increasing lens strength is not due to inability to discriminate the stimuli. but is related to central processing competition between eyes under dichoptic conditions. Binocular perception is not merely the summation of information from two receptor channels. Furthermore. theories of dominance in binocular vision which simply assign weights to the two eyes (Levelt. $1968)$ must acknowledge image clarity as a variable in assigning the weights. While it might be argued that the volume of information in the monocular condition has also been reduced. and this accounts for the superior performance, a study by Sampson and Horrocks (1967) indicates this is not the case. Using overlapped number pairs which could be presented either monocularly or dichoptically. they demonstrated that recall was more accurate when both numbers were presented monocularly than when they were presented dichoptically: even though the percepts were identical. This study does not allow speculation on the stability or maintenance of the dominance change induced or on the effect of eves of unequal acuity upon dominance. However. the measurement of dominance by the dichoptic recall procedure and the ease of manipulation of the obtained dominance have implications for use in disorders where dominance is a known (amblyopia) or presumed (dyslexia) factor.

\section{REFERENCES}

Adler. F.. \& Tal. E. The role of eye dominance in perception in hemiplegic patients. Archives of Physical Medicine \& Rehabilitation. 1966. 47.67-71.

Berner. G. E.. \& Berner. D. F. Relation of ocular dominance. handedness. and the controlling eye in binocular vision. Archives of Ophthalmology. 1953. 50. 603-608.

Crovitz. H. F. Differential acuity of the two eyes and the problem of ocular dominances. Science. 1961.134.614.

Gahagan. L. Visual dominance-acuity relationships. Journal of General Psychology. 1933. 9. 455-459.

Gronwall. D. 11. A.. \& Sampson. H. Ocular dominance: A test of two hypotheses. British Journal of Psychology. 1971, 62. 175-185.

Lederer. J. Ocular dominance. Australian Journal of Optonetry. $1961.44 .531-539 \mathrm{ff}$.

Levelt. W. J. Y. On binocular rivalry. The Hague: Mouton. 1968.

Merrell. D. J. Dominance of eye and hand. Human Brology. 1957. 29, 314-328.

Perry. N. W.. Jr.. \& Childers. D. G. Monocular contribution to binocular vision in normals and amblyopes. In G. B. Arden (Ed.). The lisual sistem: leurophysiolog?. biophlysics, and their clinical applications. New York: Plenum Press. 1972.

Sampson. H.. \& Horrocks. J. B. Binocular rivalry and immediate memory: Quarterl! Journal of Experimental Psychology. 1967. 19. 224-231.

Walls. G, L. A theory of ocular dominance. Archives of Ophthalmology. 1951, 45.387-412.

(Received Iuly 17.1972:

revision receired October 10.1972 .1 